\title{
HUMAN FACTORS RESEARCH FOR SPACE EXPLORATION: MEASUREMENT, MODELING, AND MITIGATION
}

\author{
Mary K. Kaiser, NASA Ames Research Center (Organizer and Chair) \\ Christopher S. Allen, NASA Johnson Space Center \\ Immanuel Barshi, NASA Ames Research Center \\ Dorrit Billman, San Jose State University/NASA Ames Research Center \\ Kritina L. Holden, Lockheed Martin/NASA Johnson Space Center
}

As part of NASA's Human Research Program, the Space Human Factors Engineering Project serves as the bridge between Human Factors research and Human Spaceflight applications. Our goal is to be responsive to the operational community while addressing issues at a sufficient level of abstraction to ensure that our tools and solutions generalize beyond the point design. In this panel, representatives from four of our research domains will discuss the challenges they face in solving current problems while also enabling future capabilities.

Historically, engineering-dominated organizations have tended to view good Human Factors (HF) as a "desirement" rather than a requirement in system design and development. Our field has made significant gains in the past decade, however; the Department of Defense, for example, now recognizes Human-System Integration (HSI), of which HF is a component, as an integral part of their divisions' hardware acquisition processes. And our own agency was far more accepting of HF/HSI requirements during the most recent vehicle systems definition than in any prior cycle.

Nonetheless, HF subject matter experts at NASA often find themselves in "catch up" mode... coping with legacy systems (hardware and software) and procedures that were designed with little regard for the human element, and too often with an attitude of "we can deal with any operator issues during training." Our challenge, then, is to segregate the true knowledge gaps in Space Human Factors from the prior failures to incorporate best (or even good) HF design principles. Further, we strive to extract the overarching core HF issues from the point-designspecific concerns that capture the operators' (and managers') attention.

Generally, our approach embraces a "3M" approach to Human Factors: Measurement, Modeling, and Mitigation. Our first step is to measure human performance, to move from subjective anecdotes to objective, quantified data. Next we model the phenomenon, using appropriate methods in our field, modifying them to suit the unique aspects of the space environment. Finally, we develop technologies, tools, and procedures to mitigate the decrements in human performance and capabilities that occur in space environments. When successful, we decrease risks to crew safety and to mission success. When extremely successful (or lucky), we devise generalizable solutions that advance the state of our practice.

Our panel is composed of researchers from diverse domains of our project... from different boxes, if you will, of the Human Factors Analysis and Classification System (HFACS) 
framework (Shappell \& Wiegmann, 2001). Yet there is more that unites us than divides us, both in terms of our methodological approach and the satisfaction derived from our modest role in enabling the exploration of our final frontier. Following are summaries our panelists’ presentations:

\title{
THE PHYSICAL ENVIRONMENT: MODELING SPACECRAFT ACOUSTICS
}

\author{
Christopher S. Allen
}

It is important to control acoustic levels in human space flight vehicles and habitats to protect crew hearing, allow for voice communications, and to ensure a healthy and habitable environment in which to work and live. Design and development of quiet space vehicles is a difficult problem because of the large number and variety of noise sources and propagation paths, and also because of the significant vehicle complexity. Acoustic modeling can play an important role in the design and development of human space flight vehicles to determine component source allocations, energy propagation paths, the need for specific noise controls, and to determine the amount of reverberation in the habitable volume. Also, acoustic models can be used to assist with the development and implementation of spaceflight acoustic materials and to predict their effectiveness including sound containment, absorption and vibration isolation.

These models can then be updated later in the design cycle to use measured sound power levels of the actual noise sources as inputs, thus producing a more refined and accurate model. Acoustic modeling can take several forms, including analytical and empirical methods. But in recent years, computer-based modeling has become sophisticated and easier to implement for complicated geometries. These numerical methods include Statistical Energy Analysis (SEA), Finite Element (FE) method, Boundary Element Method (BEM), and Ray Tracing methods (von Estorff, 2007). In order to illustrate the use of acoustic modeling, an example of a simplified space vehicle environment, modeled using SEA, will be compared with validation measurements in a representative acoustic mockup.

\section{THE TECHNOLOGY ENVIRONMENT: HUMAN-SYSTEM INFORMATION EXCHANGE IN NEXT-GENERATION SPACE SYSTEMS}

\author{
Kritina L. Holden
}

Designers of modern spacecraft face many challenges in the area of human-system information exchange. Astronaut performance depends heavily on the intuitiveness and ease of use of the displays and controls with which they have to interact during a mission. Furthermore, crews will often have to use the same displays and controls to perform a wide variety of tasks under dramatically different conditions. These conditions include: 1) Acceleration: one-G for pre-launch and post-landing; hyper-G for launch, micro-G for on-orbit operations, and hypo-G for planetary surface explorations; 2) Vibration: launch, entry, and abort vibration; and 3) Pressurization: pressurized and unpressurized suited and unsuited operations. Design solutions must be flexible enough to support optimal performance under all conditions, given that weight and volume constraints typically preclude unique solutions for each condition. 
Moreover, display/control real estate is typically very limited. Modern spacecraft will feature primarily glass-based interfaces, making much more information available than was available in the past. Even the majority of the control interfaces (e.g., knobs, switches, dials) will be electronic. The combination of more electronic information, and less real estate on which to display it, places a heavy premium on effectively defining and validating overall display organization and arrangement. In addition, spacecraft programs are rarely funded sufficiently to have the luxury of state-of-the-art, radiation-hardened display technologies. Thus, designers are faced with many constraints, challenges, lesser technology solutions, and high expectations from crew.

NASA is currently funding research in the area of Human-System Information Exchange in order to begin addressing some of these challenges. A few key areas of investigation are:

Cursor Control Device Design: design of a custom device for interacting with cockpit displays that works well during the high-G and vibration environment of launch, as well as the microgravity environment on orbit (Sándor \& Holden, 2008).

Readability of Displays under Vibration: research and development of human performance requirements for reading electronic displays under launch-like vibration (Adelstein, Beutter, Kaiser, McCann, Stone, Anderson, Renema, \& Paloski, 2009).

Pressurized Gloved Dexterity: investigation of how unpressurized and pressurized space gloves affect ability to interact with hand controllers, cursor control devices, and controls, knobs, and switches in the cockpit.

\section{THE TECHNOLOGY ENVIRONMENT: HUMAN-AUTOMATION TEAMING}

\section{Dorrit Billman}

Contemporary work in socio-technological systems is primarily information work, concerning forming, assessing, and making decisions. This work depends on the interaction among multiple humans and a variety of supporting tools. The coordination of decision making - at multiple levels - is frequently the most critical and difficult aspect of the interaction. Despite the importance of interaction, primary design attention may be spent on system components, from selection and training of workers to the algorithms of a smart device, with interaction left to "emerge" as an after effect. Indeed, design of sound interaction can be expensive and time consuming.

NASA is committed to making interaction a focal part of design across its many socio-technical systems. One focus within NASA's Space Human Factors Engineering is developing tools and methods to reduce the costs of designing sound interaction. By "design of interaction" we include the whole cycle of identifying needs, generating a design, prototyping, implementation, assessment, and iteration over these phases (Wharton, Rieman, Lewis, \& Polson, 1994). One aspect of our work is identifying additional activities at any point in the design cycle that might be computer-supported by delegating (or automating) component functions. Our research 
identifies and develops such tools and methods, with the accompanying goal of delimiting the conditions for which particular approaches are most useful. Rather than focusing on solving problems in a particular application, we seek generalizeable tools and methods.

However, the process of developing generalizeable tools should be grounded by close familiarity with specific cases, and a core case in our current work is one of the Mission Control groups, Attitude Determination and Command Officer (ADCO). In coordination with Russian counterparts, the ADCO group is responsible for the movement of the International Space Station (ISS). We are studying their planning process for specifying the sequence of activities needed to control the orientation and movement of the ISS.

I will comment on claims developing from two aspects of our work in the ADCO planning domain: need-identification and assessment. Regarding need identification, we claim that neither constraint-driven analysis (as developed in Work Analysis and focused on control of physical systems) nor task analysis at the level of particular action sequences (as outlined in many approaches descended from Hierarchical Task Analysis) is the best approach. I will summarize a) our "middle way" approach, b) some of the tools and methods we are developing, and c) the conditions when our approach may be most helpful.

Regarding evaluation, we claim that focus on errors may not be the most productive evaluation metric for this case, but rather resource metrics can best identify the limiting factors of design. These resource metrics do include metrics for performance time on standard tasks. However, particularly important resource metrics are learning measures such as time and stability of basic learning, and ability to transfer to never-taught conditions including novel domain problems, and novel work demands such as interruptions or collaborations. I will summarize a) metrics under consideration, b) an empirical assessment, and c) the conditions when such metrics may be most helpful.

\section{TRAINING FOR THE LONG RUN: CHALLENGES OF LONG-DURATION MISSIONS}

\section{Immanuel Barshi}

Ground-based pre-flight training and in-space just-in-time training and task rehearsal will play critical and complementary roles for exploration missions. Because long-duration missions preclude the possibility of easily providing new crew members from the ground who have been specially trained on specific emerging problems, new tasks and new scientific or mission operations, crew members will have to be able to address all known and emerging mission needs. To meet that challenge, on-board training systems will have to enhance the autonomy and effectiveness of exploration crews. We will continue to depend on the deep knowledge astronauts acquire of the idiosyncrasies of the flight systems they live with and the tasks they have to perform. However, given the nature of the missions, onboard training opportunities for individuals and teams will be necessary, such as in reconfigurable training and mission rehearsal systems. These systems will enable the crews to keep their skill levels up to par and to develop new skills or practice new procedures to resolve new challenges as they arise. 
Increasing communication delays between crews and ground support mean that astronauts need to be prepared to handle the unexpected on their own. As crews become more autonomous, their potential span of control and required expertise is much larger than in current missions. It is not possible to train for every eventuality ahead of time on the ground or maintain such skills across long intervals of disuse. New training approaches must be skill-based rather than task-based, emphasizing the acquisition of general skills such as avionics trouble-shooting, or even broader skills such as creative problem solving (Baer, 1996).

Furthermore, a team of experts is not necessarily an expert team. Thus, team training will be particularly important, and especially so for multicultural and international crews on longduration missions. Research in many other high-risk domains (e.g., aviation, the military, nuclear power and medicine) shows that effective teamwork can provide resilience in the face of challenging problems. The same is true for the people of Launch and Mission Control, particularly as mission complexity increases and resources available for training decrease.

\section{REFERENCES}

Adelstein, B. D., Beutter, B. R., Kaiser, M. K., McCann, R. S., Stone, L. S., Anderson, M. A., Renema, F., and Paloski,

W. H. (2009). Influence of Combined Whole-Body Vibration Plus G-Loading on Visual Performance. NASA Technical Memorandum 2009-215386.

Baer, J. (1996). The effects of task-specific divergent-thinking training. Journal of Creative Behavior, 30, 183-187.

Sándor, A., \& Holden, K. (2008). Cursor Control Device Test Battery: Development and Application. HFES Conference, September 2008, New York, NY.

Shappell, S.A. \& Wiegmann, D.A. (2001). Applying Reason: The Human Factors Analysis and Classification System (HFACS). Human Factors and Aerospace Safety, 1(1), 59-86.

von Estorff, O. (2007). Numerical methods in acoustics: Facts, fears, and future. 19th International Congress on Acoustics, September 2007, Madrid.

Wharton, C., Rieman, J., Lewis, C., \& Polson, P. (1994). The cognitive walkthrough method: A practitioner's guide. In J. Nielsen \& R. L. Mack (eds.). Usability inspection methods. New York, NY: John Wiley. 